Itinerarios culturales y rutas turístico-culturales 15

\title{
Para saber más. Bibliografía especializada sobre itinerarios y rutas
}

Antonio-José Campesino Fernández. Catedrático de Análisis Urbano y Regional. Universidad de Extremadura. Vicepresidente de ICOMOS-España

(a $n, y, y 0$ a

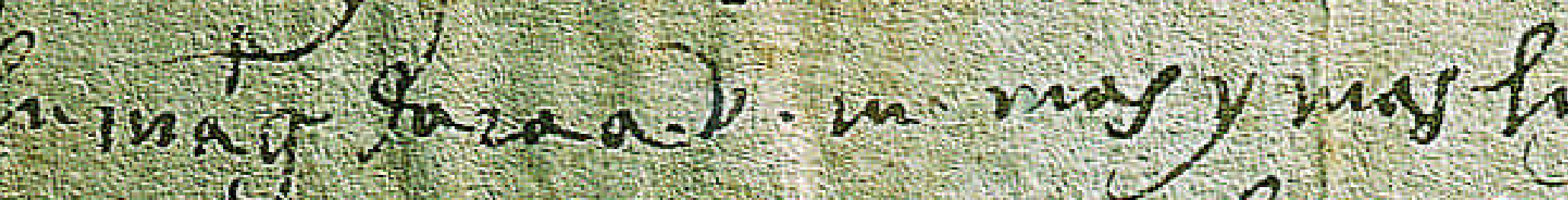

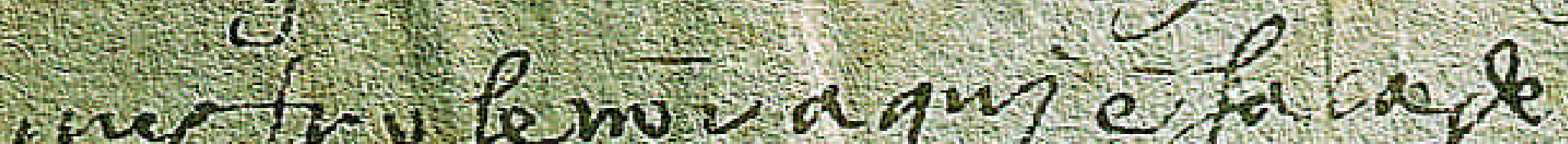

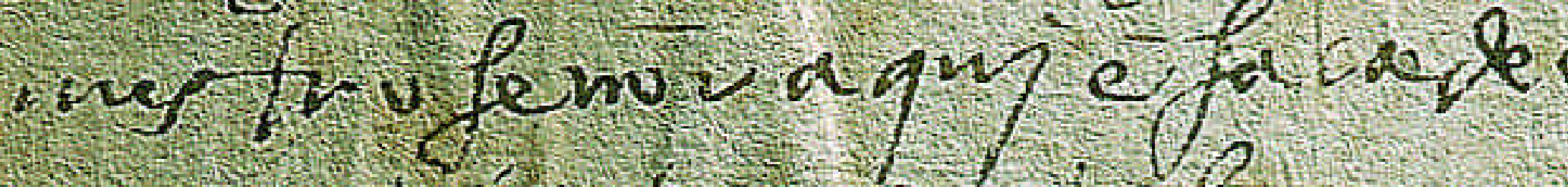

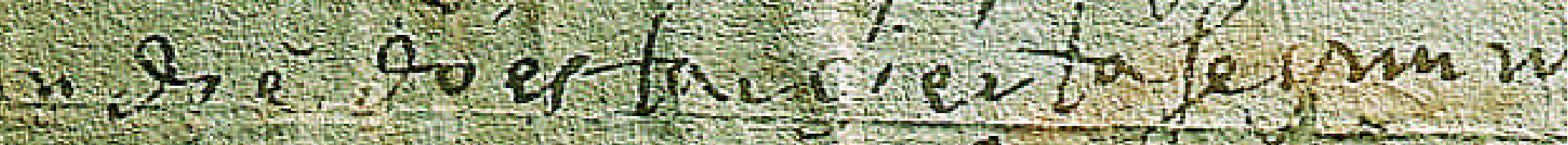

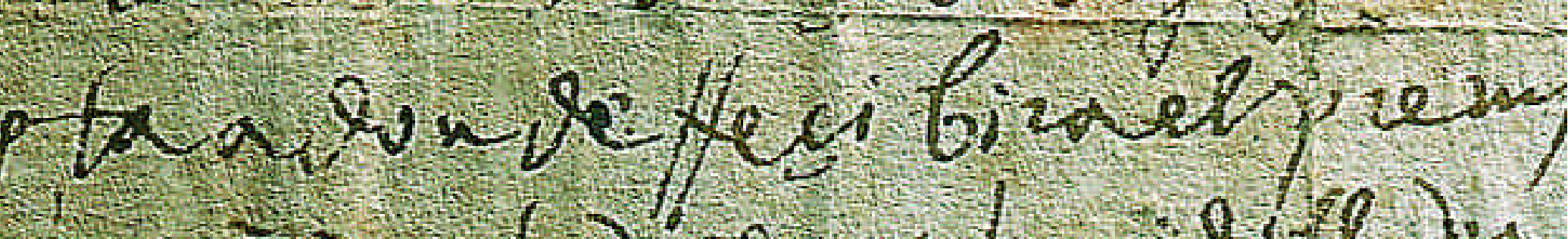

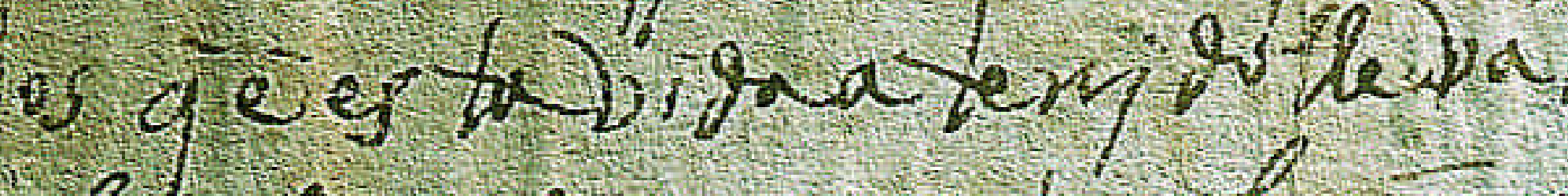
ti $p$ l $p$. u

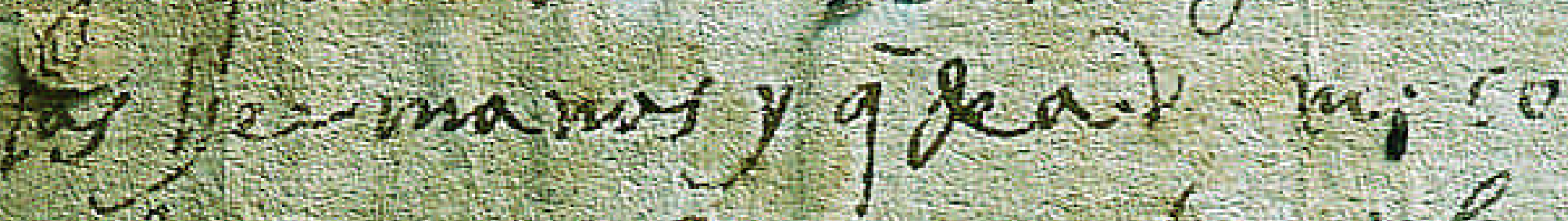


Recorrer a través de la bibliografía existente itinerarios y rutas del patrimonio cultural es una tarea posible a partir de la numerosa producción especializada, que se puede encontrar tanto en monografías y publicaciones periódicas. A continuación se of rece un listado de referencias principalmente de escala nacional, en el que aparecen recogidos proyectos turísticos específicos -como el Itinerario Cultural de Almorávides y Almohades del Legado Andalusí, la Red de Juderías de España-Camino de Sefarad o el Camino de Santiago francés, por poner algunos ejemplos- 0 análisis más generales de las dinámicas y repercusiones de estas rutas e itinerarios en sus territorios.

AA.VV. Los Itinerarios como Patrimonio Cultural. UNESCO, Centro de Documentación, Madrid, 1994

AA. VV. Caminos de Sefarad. Cáceres, Córdoba, Girona, Hervás, Ribadavia, Segovia, Toledo, Tudela. Consejería de Industria y Turismo de la Junta de Extremadura, Mérida, 1995

AA. VV. La Península Ibérica y las Rutas Culturales del Mediterráneo: comercio y civilización; un ámbito intercontinental. Alicante, 1997

AA. VV. La herencia judía en la Red de Juderías de España-Caminos de Sefarad. Mercados emisores y especialización del turismo urbano. Girona, 2000

AA. VV. Actas del Congreso Internacional de Itinerarios Culturales. Ministerio de Educación, Cultura y Deporte, Madrid, 2001

AA. VV. El Patrimonio Intangible y otros aspectos relativos a los Itinerarios Culturales [Actas del Congreso Internacional, 20 a 24 de junio]. Gobierno de Navarra, Pamplona, 2002

Q Manuscrito de Santa Teresa. El Camino de la Lengua Castellana recorre lugares donde realizaron su obra o parte de ella escritores que impulsaron el desarrollo de la lengua y literatura castellanas, como Santa Teresa de Jesús en Ávila / Fundación Camino de la Lengua Castellana
AA. VV. Rutas culturales y turísticas del patrimonio industrial [Actas de las VI Jornadas del Patrimonio Industrial. Gijón, octubre de 2004]. Asociación Arqueológica Industrial, Patrimonio Cultural y Natural (INCUNA), Colección "Los ojos de la memoria", 4. Gijón, 2005

AA. VV. Itinerario Cultural de Almorávides y Almohades. Fundación El Legado Andalusí

ABEJÓN, T.; FERNÁNDEZ, E. y MALDONADO, A. "Fuentes de información especiali zadas en Patrimonio Arquitectónico existentes en Internet". En IGLESIAS GIL, J. M. (Ed.). Cursos sobre el Patrimonio Histórico 4 (13 a 16 de julio, 1999). Actas de los X Cursos Monográficos sobre el Patrimonio Histórico. Universidad de Cantabria, Ayuntamiento de Reinosa, 2000, pp. 351-384

ALONSO ORTEGA, J. L. "El Canal de Castilla: atractiva e importante ruta turística en tierras castellanas". Revista de Estudios Turísticos, 112. Instituto de Estudios Turísticos, Madrid, 1988, pp. 55-79

ÁlVAREZ MORA, A. (Dir.). Territorio y Patrimonio. Revista Ciudades, 4. Instituto de Urbanística de la Universidad de Valladolid, Valladolid, 1998

AMENDOEIRA, A. P. "Las cañadas de la trashumancia en la Península Ibérica: un verdadero itinerario cultural". En AA. VV. El Patrimonio Intangible y otros aspectos de los Itinerarios Culturales. Gobierno de Navarra, Pamplona, 2002, pp. 171-173

AMENDOEIRA, A. P. "Reflexión sobre la categoría de paisaje cultural en la lista del Patrimonio Mundial de las Américas". En La Representatividad en la Lista del Patrimonio Mundial. El Patrimonio Cultural y Natural de Iberoamérica, Canadá y Estados Unidos. Memorias. Conaculta. INAH. Dirección de Patrimonio Mundial e ICOMOS, Santiago de Querétaro, 2004, pp. 77-80

ANDRÉS ORDAX, S. "El Duero castellano: posibilidades culturales". En PARRADO DEL OLMO, J. M. (Coord.).La cultura como elemento de unión en Europa. Rutas culturales activas. Fundación del Patrimonio Histórico de Castilla y León, Burgos, 2002, pp. 185-219

ANTÓN CLAVÉ, S. Rutas e itinerarios culturales. Propuestas y desafíos. Karis (Observatorio Europeo de Políticas Culturales, Urbanas y Territoriales), 8. Barcelona, 1988, pp. 11-18

ANTÓN CLAVÉ, S. "Turismo, territorio y cultura". En MORALES MATOS, G. (Ed.). Actas Turismo y Ciudad. Grupo de Geografía Urbana de la Asociación de Geógrafos Españoles y Excmo. Ayuntamiento de Las Palmas de Gran Canaria, Las Palmas de G. C., 2000, pp. 29-41

AYUNTAMIENTO DE CARM ONA. Ruta Bética Romana (RUBERO). Proyecto Europeo. Oficina de la Bética Romana, Carmona 
X La biblioteca del Monasterio de Silos (Burgos) guarda en la actualidad más de 60.000 volúmenes, entre ellos, incunables, códices y pergaminos / Fundación CAMINo DE LA Lengua Castellana

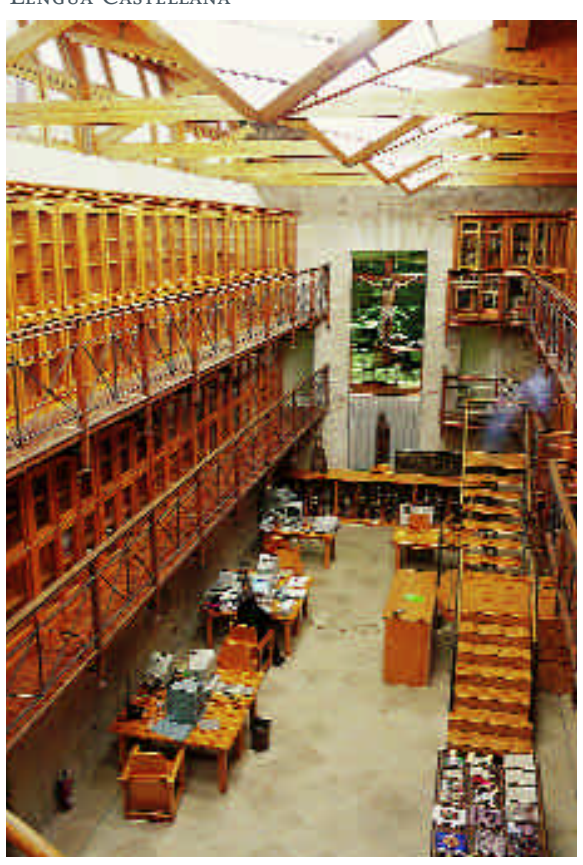

BELLIDO GANT, Mạ. L. "Expectativas de la virtualización de los itinerarios culturales". En PARRADO DEL OLMO, J. M. (Coord.). La cultura como elemento de unión en Europa. Rutas culturales activas. Fundación del Patrimonio Histórico de Castilla y León, Burgos, 2002, pp. 367-388

BERNAL SANTA OLALLA, B. "El conocimiento del territorio como base fundamental en la aventura de definir caminos o itinerarios culturales". En Actas del VI Congreso Internacional Italia-España, 2002. T. I. Madrid, 2004

BORRÁS GUALÍS, G. "Las rutas del mudejarismo aragonés". En PARRADO DEL OLMO, J. M. (Coord.). La cultura como elemento de unión en Europa. Rutas culturales activas. Fundación del Patrimonio Histórico de Castilla y León, Burgos, 2002, pp.107-136

CABERO DIÉGUEZ, V. "Paisajes rurales y culturales: conservación, educación y desarrollo". En RAMOS CASTELLANOS, P. (Ed.). Medio Ambiente: calidad ambiental. Ediciones de la Universidad de Salamanca, Aquilafuente, 32. Salamanca, 2002, pp. 213-240

CAMPESINO FERNÁNDEZ, A.-J. "Los Caminos de Santiago: ciudades y recursos turís ticos". En TORRES, Mạ. P.; PÉREZ, A.; LOIS, R. C. (Eds.). Los Caminos de Santiago y el territorio. Xunta de Galicia, Santiago, 1993, pp. 667-712

CAMPESINO FERNÁNDEZ, A.-J. "Territorio de frontera: adaptación, trazado y estructura en la raya española". En ROSSA, W.; ARAUJO, R. y CARITA, H. (Coords.). Colóquio Internacional. A Cidade como Civilização: Universo Urbanístico Português, 1415-1822. Comissão Nacional para as Comemorações dos Descobrimentos Portugueses Lisboa, 2001, pp. 751-767

CAMPESINO FERNÁNDEZ, A.-J. "El paisaje: patrimonio cultural". En GÓMEZ DE LA IGLESIA, R. (Dir.). Cultura, Desarrollo y Territorio. III Jornadas sobre Iniciativa Privada y Sector Público en la Gestión de la Cultura. XABIDE, Gestión Cultural y Comunicación, Vitoria-Gasteiz, 2001, pp. 227-239

CAMPESIN O FERNÁNDEZ, A.-J. (Dir.). Territorio y Ciudades Abaluartadas en la Raya Ibérica: de Frontera a Paisaje Cultural de la Humanidad. (Proyecto de Investigación en el que se integran los Departamentos de Geografía de las Universidades de Santiago, León, Salamanca, Extremadura y Sevilla). Cáceres, 1981-2005

CANTARERO QUESADA, J. Mạ. (Coord.). Turismo y cultura en el medio rural. Gestión sostenible y competitiva. Junta de Andalucía, Consejería de Turismo y Deporte, Jaén, 2003

CAÑIZARES RUIZ, Mạ. C. "Turismo de interior en Castilla-La Mancha: Ruta de D. Quijote y Ruta de los Caballeros". En VALENZUELA RUBIO, M. (Coord.). Los turismos de interior. El retorno a la tradición viajera. Universidad Autónoma de Madrid, AGE y Universidad de Castilla-La Mancha, Madrid, 1997, pp. 745-749

CAPEL SÁEZ, H. "Las Rutas Culturales como Patrimonio de la Humanidad. El caso de las fortificaciones americanas del Pacífico". En Biblio3W. Revista Bibliográfica de Geografía y Ciencias Sociales (Serie documental de Geo-Crítica). Vol. X, no 562, 30 de enero. Universidad de Barcelona, Barcelona, 2005

CELDRÁN GOMÁRIZ, P. Red de Juderías de España. Caminos de Sefarad. Alymar, 2005

DE CASTRO, J.; COBOS, F.; CORTES, J. L. y CUADRADO, A. Guía de la Ruta de las Fortificaciones de Frontera-Salamanca. Fundación del Patrimonio Histórico de Castilla y León, Salamanca, 2001

DE LA IGLESIA, M. A.; ÁLVAREZ, D.; GONZÁLEZ, J. y TUSET, F. Plan Director El Cid en Vivar y Camino del Destierro (Ego Ruderico). Excma. Diputación Provincial de Burgos, Burgos, 1997

FAUSTO BARONE, A. "El influjo del Islam en el sur de Italia. Su papel en los itinerarios culturales". En PARRADO DEL OLMO, J. M. (Coord.). La cultura como elemento de unión en Europa. Rutas culturales activas. Fundación del Patrimonio Histórico de Castilla y León, Burgos, 2002, pp. 87-106

FERnÁNDEZ TABALES, A. (Dir.). Manual de Gestión Local del Turismo. Directrices para Agentes Públicos de la Provincia de Sevilla. Turismo de la Provincia, Diputación de Sevilla, Sevilla, 2004 
FUNDACIÓN DEL PATRIM ONIO DE CASTILLA Y LEÓN. "Ruta de las Fortificaciones, punto de encuentro". Revista Patrimonio Histórico de Castilla y León, 3, Valladolid 2000 , pp. $5-8$

FUNDACIÓN DEL PATRIMONIO DE CASTILLA Y LEÓN. "Ruta Arqueológica por los Valles de Zamora. Un recorrido por el pasado". Revista Patrimonio Histórico de Castilla y León, 5, Valladolid, 2001, pp. 5-9

GARCÍA DE MIGUEL, J. Ma “ "Itinerarios culturales relacionados con el patrimonio histórico minero". En AA. VV. El Patrimonio Intangible y otros aspectos relativos a los Itinerarios Culturales. Gobierno de Navarra, Pamplona, 2002, pp 145-157

GARCÍA GRINDA, J. L. “El Camino de Santiago: Conjunto Histórico Territorial. Patrimonio, Planeamiento Urbanístico y Gestión". En LÓPEZ TRIGAL, L. (Dir.) Ciudades y Villas Camineras Jacobeas. III Jornadas de Estudio y Debate Urbanos. Universidad de León, León, 2000, pp. 113-132

GARCÍA OTERO, J. Mạ. "Los medios de comunicación y su papel en el conocimiento de las rutas culturales". En PARRADO DEL OLMO, J. M. (Coord.). La cultura como elemento de unión en Europa. Rutas culturales activas. Fundación del Patrimonio Histórico de Castilla y León, Burgos, 2002, pp. 303-317

GARCíA ZARZA, E. "La Ruta del Castellano". En MORALES MATOS, G. (Ed.). Actas Turismo y Ciudad. Grupo de Geografía Urbana de la Asociación de Geógrafos Españoles y Excmo. Ayuntamiento de Las Palmas de Gran Canaria, Las Palmas de G. C., 2000, pp. 45-53

GARCÍA, M. y GRANDE, J. La Cañada Soriana Occidental. El País-Aguilar, Madrid, 2001

GÓMEZ DE LA IGLESIA, R. (Dir.). Cultura, Desarrollo y Territorio. III Jornadas sobre Iniciativa Privada y Sector Público en la Gestión de la Cultura. XABIDE, Gestión Cultural y Comunicación, Vitoria-Gasteiz, 2001

GÓMEZ, J. y G-QUIJANO, C Rutas e itinerarios turísticos en España. Síntesis Madrid, 1991

GÓNGORA, A.; LOVILLO, I.; PERALTA, C. y VALDÉS, A. (Eds.). Guía de Rutas Culturales de Andalucía. Junta de Andalucía, Consejería de Turismo, Comercio y Deporte, Turismo Andaluz, S.A., Sevilla, 2004

GRANDE IBARRA, J. (Ed.). Actas del Congreso Europeo sobre Itinerarios Culturales y Rutas Temáticas [20 a 22 de noviembre de 1997]. Fundación Caja Rioja, Logroño, 1998

GRANDE IBARRA, J. "Los itinerarios culturales, la generalización de un producto". En I Congreso de Turismo Cultural, Madrid, 2002

HELGUERA, J.; TAPIA, N. G. y MOLINERO, F. El Canal de Castilla. Junta de Castilla y León, Valladolid, 1988

HORTELANO MÍNGUEZ, L. A. "El desarrollo del turismo cultural en el medio rural de Castilla y León". En CANTARERO QUESADA, J. Mạ. (Coord.). Turismo y cultura en el medio rural. Gestión sostenible y competitiva. Junta de Andalucía, Consejería de Turismo y Deporte, Jaén, 2003, pp. 77-113

HUMBERT, A. "Patrimonio cultural y geografía. Los paisajes culturales". En AA. VV. Jornadas sobre patrimonio cultural: un enfoque pluridisciplinar. Departamento de Educación y Cultura. DGA. Zaragoza, 1998

IBÁÑEZ PÉREZ, A. “El Camino de Santiago en España: una realidad consolidada”. En PARRADO DEL OLMO, J. M. (Coord.). La cultura como elemento de unión en Europa. Rutas culturales activas. Fundación del Patrimonio Histórico de Castilla y León, Burgos, 2002, pp. 57-83

ICOMOS. Simposio Internacional sobre Encrucijadas Culturales Intercontinentales, Itinerarios Culturales, Legislación y Turismo Cultural [5 a 8 de septiembre]. CIIC, Santa Cruz de Tenerife, 1998

ICOM OS. Congreso Internacional sobre Fortificaciones Abaluartadas Hispano Portuguesas a través del mundo [18 a 20 de mayo]. CIIC, Ibiza, 1999
ICOMOS. Actas de las I Jornadas de Expertos en Protección y Promoción de Bienes Culturales sobre el Itinerario Cultural de la Vid y del Vino de los Pueblos del Mediterráneo [17 a 19 de mayo]. Gobierno de la Rioja, Santo Domingo de la Calzada, 1999

ICOMOS. Seminario sobre Metodología, Definiciones y Aspectos Operativos de los Itinerarios Culturales. CIIC, Ibiza (21 y 22 de mayo, 1 a parte) y Mexico D.F. y Guanajuato (2ㅇ parte), 1999

\section{ICOMOS. El Camino Real Intercontinental. CIIC, La Laguna, 2001}

ICOMOS. 1. Patrimonio Intangible e Itinerarios Culturales en un contexto Universal; 2. Primeros pasos para la elaboración de un Pre-Inventario de Itinerarios Culturales: a) Estrategias y equipos; b) Proyectos y contenidos. CIIC, 2001

ICOMOS. Reunión Científica sobre la independencia conceptual y sustantiva de los itinerarios culturales respecto a los paisajes culturales [4 de diciembre]. CIIC, Madrid, 2002

ICOMOS. Reunión de Expertos en Itinerarios Culturales: Definición, contenidos metodología y operatividad [17 a 22 de mayo]. CIIC, Madrid, 2003

ICOMOS. Ideas Preliminares para el establecimiento de una Carta Internacional de Itinerarios Culturales [29 a 30 de octubre]. CIIC, Victoria Falls, Zimbabwe, 2003

ICOMOS. Encuentro Científico Internacional sobre Itinerarios Culturales. Identificación, Promoción e Inventario de los Itinerarios Culturales. Fortificaciones, puertos y ciudades en la estructura de los Itinerarios Culturales. Rutas de Comercio, Control del Territorio y Peregrinaje [El Ferrol, 1, 2 y 3 de octubre de 2004]. Comité Nacional Español de ICOMOS, X, Ministerio de Cultura, Icomos-España, Concello de Ferrol y Xunta de Galicia, 2005

IRURTIA, V. "Rutas e itinerarios culturales". En I Congreso de Turismo Cultural, Madrid, 2002

JANER AMARGÓS, E. "Rutas Culturales catalanas y su relación con el Ebro catalán y aragonés - El Palau Robert. Centro de Información de Cataluña". En PARRADO DEL OLMO, J. M. (Coord.). La cultura como elemento de unión en Europa. Rutas culturales activas. Fundación del Patrimonio Histórico de Castilla y León, Burgos, 2002, pp. 171-183

JUNTA DE CASTILLA Y LEÓN. Ruta Vía de la Plata por Castilla y León. Sotur, S.A. Consejería de Industria, Comercio y Turismo, Salamanca, 2002

JUNTA DE EXTREMADURA. Ruta Vía de la Plata por Extremadura. Consejería de Obras Públicas y Turismo, Mérida, 2001

KREMEZI, C. “Le Projet 'Itinaires culturels du sud-est europeen'. Participation et proposition greque“. En MARTORELL CARREÑO, A. (Coord.). ICOMOS. XIII Asamblea General. Estrategias relativas al Patrimonio Cultural Mundial. Simposio Científico. La Salvaguarda en un Mundo Globalizado. Principios, Prácticas y Perspectivas [Madrid, 1 a 5 de diciembre, 2002]. Comité Nacional Español de ICOMOS, Madrid, 2002, pp. 34-36

LOIS, R. C. y SANTOS, X. M. "El Camino de Santiago". En AA. VV. La Actividad Turística Española en 1998. Asociación Española de Expertos Científicos en Turismo (AECIT), Madrid, 1999, pp. 597-603

LÓPEZ CARRETÓN, J. Real Fuerte de la Concepción de Aldea del Obispo (Salamanca). Asociación Cultural El Fuerte. $2^{a}$ edición, Salamanca, 1999

LÓPEZ OLIVARES, D. La ordenación y planificación integrada de los recursos territoriales turísticos. Servicio de Publicaciones, Universitat Jaume I, Castellón, 1998

LÓPEZ PALOMEQUE, F. "Política turística y territorio en el escenario de cambio turístico". En Boletín de la Asociación de Geógrafos Españoles: Cambio Turístico y nuevos procesos territoriales, 28. AGE, Murcia, 1999, pp. 23-38

LÓPEZ PALOMEQUE, F. “El patrimonio y las nuevas formas de turismo: ciudades y villas camineras jacobeas". En LÓPEZ TRIGAL, L. (Dir.). Ciudades y Villas Camineras 
Jacobeas. III Jornadas de Estudio y Debate Urbanos. Universidad de León, León, 2000, pp. 51-69

LÓPEZ, F. y GÓMEZ, B. “Nuevos productos de turismo cultural: Castillos y fortalezas en Cataluña". En MORALES MATOS, G. (Ed.). Actas Turismo y Ciudad. Grupo de Geografía Urbana de la Asociación de Geógrafos Españoles y Excmo. Ayuntamiento de Las Palmas de Gran Canaria, Las Palmas de G. C., 2000, pp. 155-162

LORENTE, E.; MOROLLÓN, P.; BLANCO, J. y VÁZQUEZ, A. Rutas de Toledo. Rea Fundación Toledo. Guías Artísticas Electa, Toledo, 1995

MALO GARAOYA, L. "La fuerza del idioma: las posibilidades de la lengua como fuerza del conocimiento". En PARRADO DEL OLMO, J. M. (Coord.). La cultura como elemento de unión en Europa. Rutas culturales activas. Fundación del Patrimonio Histórico de Castilla y León, Burgos, 2002, pp. 273-301

MARCHENA GÓMEZ. M. Territorio y turismo en Andalucía. Dirección General de Turismo, Consejería de Economía y Fomento, Junta de Andalucía, Sevilla, 1987

MARCHENA GÓMEZ, M. (Ed.). Turismo urbano y patrimonio cultural. Una perspectiva europea. Turismo de Sevilla, Diputación de Sevilla. Colección Documentos 1 Sevilla, 1998

MARCHENA, M. y CARRASCO, G. "La promoción turística del patrimonio cultural en Andalucía". En VALENZUELA RUBIO, M. (Coord.). Los turismos de interior. El retorno a la tradición viajera. Universidad Autónoma de Madrid, AGE y Universidad de CastillaLa Mancha, Madrid, 1997, pp. 697-705

MARIÑAS OTERO, E. "La Vía de la Plata: itinerario turístico-cultural". Revista de Estudios Turísticos, 109. Instituto de Estudios Turísticos, Madrid, 1991, pp. 109127

MARTÍNEZ DE PISÓN, E. "El paisaje, patrimonio cultural". Revista de Occidente, nos 194-195. Madrid, 1997, pp. 37-49

MARTORELL CARREÑO, A. (Coord.). ICOMOS. XIII Asamblea General. Estrategia relativas al Patrimonio Cultural Mundial. Simposio Científico. La Salvaguarda en un Mundo Globalizado. Principios, Prácticas y Perspectivas. (Madrid, 1-5 de diciembre, 2002). Comité Nacional Español de ICOMOS, Madrid, 2002

MARTORELL CARREÑO, A. "Paisajes e itinerarios culturales: conceptos independientes que enriquecen la teoría y la práctica de la conservación cultural". The CIIC Scientific Magazine, ICOMOS, Madrid, 2004

MATA, L. M. y DE LUIS, A. Ruta de las Fortificaciones de Frontera (Ciudad Rodrigo, San Felices de los Gallegos, Aldea del Obispo y Almeida). Asociación para el Desarrollo de la Comarca de Ciudad Rodrigo (ADECOCIR), Salamanca, 2001

MIGUEL QUINTALES, L. A. Ruta del Camino Fonseca. De Salamanca a Santiago de Compostela. Amarú Ediciones, Salamanca, 2002

ORTEGA CANTERO, N. "El paisaje de España en los viajeros románticos" ERÏA, Revista de Geografía, 22. Universidad de Oviedo, Oviedo, 1990, pp. 121-137

ORTEGA VALCÁRCEL, J. "El patrimonio territorial: El territorio como recurso cultural y económico". En ÁLVAREZ MORA, A. (Dir.). Ciudades. Revista del Instituto de Urbanística de la Universidad de Valladolid.", Territorio y Patrimonio. 4 Secretariado de Publicaciones de la Universidad de Valladolid, Valladolid, 1998, pp. 33-48

PLAZA GUTIÉRREZ, J. I. "Las rutas historia-naturaleza como complemento del turismo urbano: la experiencia de Castilla y León". En PARDELLAS DE BLAS, X. X. (Dir). Estrategias Turísticas Urbanas. Asociación Galega de Ciencia Regional y Uiversidade de Vigo, Vigo, 2002, pp. 99-125

PARDELLAS DE BLAS, X.-X. (Dir). Turismo relixioso: O Camiño de Santiago. Servizo de Publicacións de la Universidade de Vigo. Vigo, 2005
PARDO RODRÍGUEZ, J. "Análisis de itinerarios y rutas culturales". Ponencia del Curso Oferta turística: diversificación y complementariedad. Fundación Cavanilles de Altos Estudios Turísticos, Benicassim, 1997

PAREJA, A.; GONZÁLEZ, A.; VÁZQUEZ, A.; VILLAVERDE, A. y SÁNCHEZ-CHIQUITO, S. Rutas turísticas de Castilla-La Mancha. Junta de Comunidades de Castilla-La Mancha, Consejería de Industria y Trabajo, Dirección General de Turismo, Comercio y Artesanía, Toledo, 1997

PARRADO DEL OLMO, J. M. (Coord.). La cultura como elemento de unión en Europa. Rutas culturales activas [Simposio Internacional, 27 a 29 de noviembre]. Fundación del Patrimonio Histórico de Castilla y León, Burgos, 2002

PATRONATO PARA LA PROMOCIÓN DEL TURISMO Y DE LA ARTESANÍA DE CÁCERES. Caminos de Sefarad. Cáceres, Córdoba, Girona, Hervás, Rivadavia, Segovia, Toledo, Tudela. Kadmos, Salamanca, 1995. S/p

PORTAS, N. "Geografia e arquitectura do territorio". En AA. VV. II Colóquio Ibérico de Geografia (Lisboa, 1980). Comunicações. Volumen II. Centro de Estudos Geográficos, Universidade de Lisboa, Lisboa, 1983, pp. 95-103

POZZANA, M. "Las rutas culturales en la Toscana: organización y sistematización". En PARRADO DEL OLMO, J. M. (Coord.). La cultura como elemento de unión en Europa. Rutas culturales activas. Fundación del Patrimonio Histórico de Castilla y León, Burgos, 2002, pp. 335-348

REVISTA GEO. El Duero. Por las orillas del río desde Urbión a Oporto. Dossier, 129. Madrid, 1997, pp. 52-88

REVISTA GEO. La ruta del idioma castellano. Dossier, 189. Madrid, 2002, pp. 56-83

REVISTA GEO. Vía de la Plata. De sur a norte, por la columna vertebral de la España romana. Dossier, 195. Madrid, 2003, pp. 54-83

RODRIGUES MOURINHO, A. “EI Duero portugués y su relación con el castellanoleonés”. En PARRADO DEL OLMO, J. M. (Coord.). La cultura como elemento de unión en Europa. Rutas culturales activas. Fundación del Patrimonio Histórico de Castilla y León, Burgos, 2002, pp. 221-235

RODRÍGUEZ RODRÍGUEZ, I. "Los municipios y el turismo cultural. La Ruta Bética Romana". En CANTARERO QUESADA, J. Ma . (Coord.). Turismo y cultura en el medio rural. Gestión sostenible y competitiva. Junta de Andalucía, Consejería de Turismo y Deporte, Jaén, 2003, pp. 285-296

RUIZ MATEOS, A. "Plan de actuación en la Vía de la Plata". En IGLESIAS GIL, J. M. (Ed.). Cursos sobre el Patrimonio Histórico 3 (21 a 24 de julio, 1998). Actas de los IX Cursos Monográficos sobre el Patrimonio Histórico. Universidad de Cantabria, Ayuntamiento de Reinosa, 1999, pp. 281-291

SÁNCHEZ ALEGRÍA, E. Ruta de La Vera de Plasencia (Río Tiétar). Barcelona, 1983

SÁEZ HIDALGO, I. El Canal de Castilla. Guía para caminantes. Junta de Castilla y León, Valladolid, 1994

SECRETARÍA DE ESTADO DE TURISMO. Rutas Turísticas. Conozca Vd. España, Madrid, 1979

SIERRA FERnÁNDEZ, Ma. P. “Camino de Santiago. Itinerario Cultural Europeo. Revitalización y contribución al desarrollo turístico del noroeste peninsular". En AA. VV. Cambios regionales a finales del siglo XX. AGE, Universidad de Salamanca, Departamento de Geografía, Salamanca, 1995, pp. 268-273

SORIA, A.; GALLEGO, M.; GARCÍA, J. L. y otros. El Camino de Santiago (I. Vías, viajes y viajeros de antaño. II. Instalaciones y señales). MOPT, Madrid, 1991-1992

SUÁREZ-INCLÁN DUCASSI, Mạ. R. “El Comité Científico Internacional de Itinerarios Culturales de ICOMOS: una apuesta para la paz y la solidaridad universal". En AA. VV. 
Actas del Congreso Internacional de Itinerarios Culturales. Ministerio de Educación, Cultura y Deporte, Madrid, 2001

SUÁREZ-INCLÁN DUCASSI, Mạ. R. “Los Itinerarios Culturales”. En La Representatividad en la Lista del Patrimonio Mundial. El Patrimonio Cultural y Natural de Iberoamérica, Canadá y Estados Unidos. Memorias. Conaculta.INAH. Dirección de Patrimonio Mundial e ICOMOS, Santiago de Querétaro, 2004, pp. 105-114

THOMAS-PENETTE, M. "Los itinerarios culturales del Consejo de Europa y las Rutas de la Seda en Europa". En España y Portugal en las rutas de la seda. Universidad de Barcelona, Barcelona, 1996

THOMAS-PENETTE, M. "Itinerarios culturales del Consejo de Europa. Importancia de paisaje. El itinerario de los parques y jardines". En AA. VV. Actas del Congreso Internacional de Itinerarios Culturales. Ministerio de Educación, Cultura y Deporte, Madrid, 2001

THOMAS-PENETTE, M. "El Camino de Santiago en Francia: El pasado y el presente". En PARRADO DEL OLMO, J. M. (Coord.). La cultura como elemento de unión en Europa. Rutas culturales activas. Fundación del Patrimonio Histórico de Castilla y León, Burgos, 2002, pp. 37-56

TORRES RIESCO, J. C. "La demanda de las rutas culturales españolas". En PARRADO DEL OLMO, J. M. (Coord.). La cultura como elemento de unión en Europa. Rutas culturales activas. Fundación del Patrimonio Histórico de Castilla y León, Burgos, 2002, pp. 239-271

TORRES, Ma. P.; PÉREZ, A.; LOIS, R. C. (Eds.). Los Caminos de Santiago y el territorio. Xunta de Galicia, Santiago, 1993

TROITIÑO VINUESA, M. A. "Patrimonio arquitectónico, cultura y territorio". En ÁLVAREZ MORA, A. (Dir.). CIUDADES, Revista del Instituto de Urbanística de la Universidad de Valladolid. Territorio y Patrimonio. 4. Secretariado de Publicaciones de la Universidad de Valladolid, Valladolid, 1998, pp. 95-104

TROTIÑ O VINUESA, M. A. "Turismo Cultural". En ANTÓN CLAVÉ, S. (Dir.). La Actividad Turística Española en 2004. Asociación Española de Expertos Científicos en Turismo (AECIT), Editorial Universitaria Ramón Areces, Madrid 2005, pp. 547-569

TURISMO DE ANDALUCÍA, S. A. Rutas Culturales. Diputación de Sevilla, Sevilla

VALDÉS FERNÁNDEZ, M. "Las rutas del mudejarismo castellano: rutas y variaciones culturales". En PARRADO DEL OLMO, J. M. (Coord.). La cultura como elemento de unión en Europa. Rutas culturales activas. Fundación del Patrimonio Histórico de Castilla y León, Burgos, 2002, pp. 137-157

VALENZUELA RUBIO, M. (Coord.). Los turismos de interior. El retorno a la tradición viajera. Universidad Autónoma de Madrid, AGE y Universidad de Castilla-La Mancha, Madrid, 1997

VEGARA CÓMEZ, A. "Cultura urbana y cultura del territorio. Los retos del urbanismo del siglo XXI". En Ciudad y Territorio/Estudios Territoriales, 95-96. Ministerio de Fomento, Madrid, 1993, pp. 245-256

VERA REBOLLO, F. J. "La variable territorial en los procesos de desarrollo turístico". En AA. VV. Arquitectura y Turismo: planes y proyectos. Centre de Recerca i Proyectes de Paisatge (CRPP) de la Universitat Politécnica de Catalunya, Barcelona, 1996, pp. 87-98

VERA, F. LÓPEZ, F.; MARCHENA, M. y ANTÖN, S. Análisis Territorial del Turismo: una nueva geografía del turismo. Ariel Geografía, Barcelona, 1997

WOLSTENCROFT, G. "Las rutas culturales inglesas: organización y puesta en desarroIlo. El ejemplo de Cornualles". En PARRADO DEL OLMO, J. M. (Coord.). La cultura como elemento de unión en Europa. Rutas culturales activas. Fundación del Patrimonio Histórico de Castilla y León, Burgos, 2002, pp. 349-366

XUNTA DE GALICIA. Obras públicas en Galicia al servicio del Camino de Santiago. Santiago, 2000

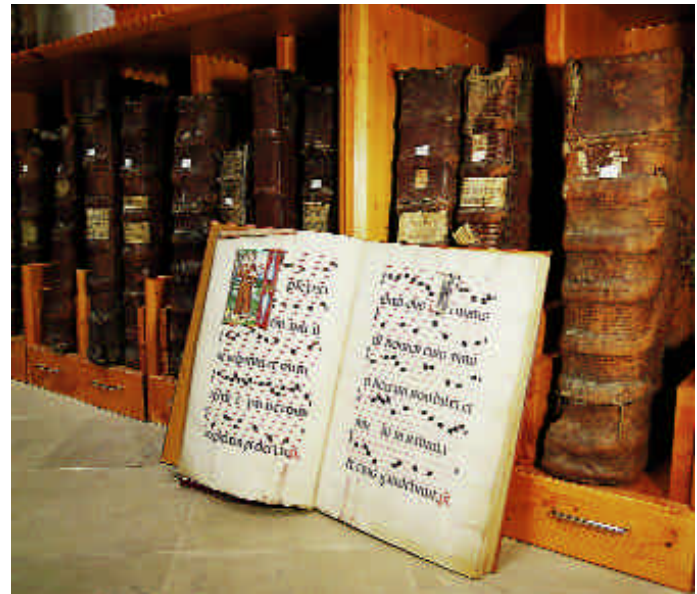

W Que la litúrgica hispánica sea hoy bien conocida se debe, en gran parte, al número y la calidad de los manuscritos litúrgicos de Silos / Fundación CAMino dE LA Lengua Castellana 